\title{
Über die differentielle Thermokraft dünner Metallschichten
}

\author{
Von E. Justi, M. Kohler und G. Lautz \\ Aus den Instituten für technische und theoretische Physik \\ der Technischen Hochschule Braunschweig
}

(Z. Naturforschg. 6 a, 456-459 [1951]; eingegangen am 12. Juli 1951)

\begin{abstract}
Der Einfluß der mittleren freien Weglänge der Elektronen auf die elektrische Leitfähigkeit, die magnetische Widerstandsänderung und auf den Wechselstromwiderstand dünner Drähte und Schichten ist Gegenstand zahlreicher Veröffentlichungen gewesen. Auf Grund theoretischer Überlegungen ist auch bei der differentiellen Thermokraft eine Abnahme mit kleiner werdender Schichtdicke zu erwarten. In der vorliegenden Arbeit wird dieser Effekt an dünnen Aufdampfschichten von spektroskopisch reinem Wismut $(99,995 \%)$ experimentell nachgewiesen. Dabei ergibt sich eine Abnahme auf $14 \%$ des Wertes am kompakten Material bei etwa $100 \AA$ Schichtdicke. Die gefundene Dickenabhängigkeit kann durch einen Einfluß der mittleren freien Weglänge der Elektronen, durch eine Vergrößerung der scheinbaren Elektronenmasse infolge einer Veränderung der Fermi-Energie bei dünnen Schichten, durch das Einwirken von Oberflächenzuständen und durch Inhomogenitäten in den Oberflächenschichten gedeutet werden.
\end{abstract}

$\mathrm{D}$ ie elektrischen Eigenschaften dünner Metallfilme zeigen beträchtliche Anomalien, wenn die Schichtdicke $d$ in die Größenordnung der mittleren freien Weglänge der Elektronen gerät. Das ist bezüglich der elektrischen Leitfähigkeit seit langem bekannt. In jüngerer Zeit wurde dieser Weglängeneffekt auch bei der magnetischen Widerstandsänderung dünner Metallschichten ${ }^{1}$ und beim anomalen Skin-Effekt ${ }^{2}$ näher untersucht. In Ergänzung zu diesen Arbeiten haben wir die experimentelle und theoretische Untersuchung des Weglängeneffektes auf die differentielle Thermokraft ausgedehnt. Die von uns begonnenen Rechnungen ergeben, daß eine energieunabhängige Weglänge $\lambda$ der Elektronen keine Schichtdickenabhängigkeit der Thermokraft bedingt ${ }^{3}$. Das erscheint plausibel, da die Thermokraft im wesentlichen einem Ausdruck der Form

$$
\frac{1}{\zeta}\left(1+\frac{1}{2} \frac{\mathrm{d} \log \lambda}{\mathrm{d} \log v}\right)_{\zeta}
$$

proportional ist, wo $\zeta$ die Fermi-Energie, und $v$ die Elektronengeschwindigkeit bedeuten. Eine Änderung der Fermi-Energie tritt bei reinem Weglängeneffekt nicht auf.

Demgegenüber darf man bei einer Abhängigkeit der Weglänge von der Energie der Elektronen auch einen Schichtdicken-Effekt bei der differentiellen Thermokraft erwarten. Setzt man etwa

$$
i=C v^{n} \quad(n>0),
$$

1 A. van Itterbeck u. A. De B ock, Ann. Physique 20,636 [1945]. so werden mit abnehmender Schichtdicke die großen Weglängen der schnellen Elektronen zunächst beeinflußt. Die Zahl der Streuprozesse mit der Oberfläche nimmt relativ zu der im Innern des Materials zu. Im Grenzfall sehr kleiner Schichtdicken werden alle Elektronen an der Oberfläche gestreut, und die Schichtdicke übernimmt die Rolle der freien Weglänge. Dann wird der zweite Summand in dem Klammerausdruck für die differentielle Thermokraft Null. Im Zwischengebiet darf man irgendeinen Wert zwischen dem des kompakten Materials und dem der extrem dünnen Schicht erwarten. Aus diesem Grunde wird man vermuten, daß die Thermokraft mit der Schichtdicke im allgemeinen abnimmt.

Die vorstehenden Úberlegungen gaben Anlaß zu Thermokraftmessungen an dünnen Wismutschichten, über die im folgenden berichtet werden soll.

\section{Die Herstellung der Schichten}

Auf Glasplatten, die mit Natronlauge und Kaliumpermanganatlösung sorgfältigst gereinigt und dann ausgeheizt worden waren, wurden gemäß Abb. 1 mit einer geeignet zurechtgeschnittenen Glimmerblende Silberelektroden aufgedampft. Als Ausgangssubstanz für die Wismutschichten diente spektroskopisch reines Material der Firma Johnson, Mat they \& Co., London, mit einem Reinheitsgrad von 99,995\%. Das Aufdampfen selbst erfolgte aus Wolframschiffchen bei schwacher Rotglut. Mit Hilfe einer zweiten Blende wurden die Wismutschichten dann so über die Silberelektroclen gedampft (Abb. 1), daß

2 G. E. H. R e u ter u. E. H. S on d he i m er, Proc. Roy. Soc. [London], Ser. A 195, 336 [1948].

3 G. L a u z , im Erscheinen. 
A und B bei einer Widerstandsmessung als Stromzuführungen und $\mathrm{C}$ und $\mathrm{D}$ als Potentialsonden dienen konnten. Für die Thermokraftmessung brachten wir bei C und D Kupfer-Konstantan-Thermoelemente an, von denen der Kupferdraht jeweils als Potentialdraht mitbenutzt wurde.

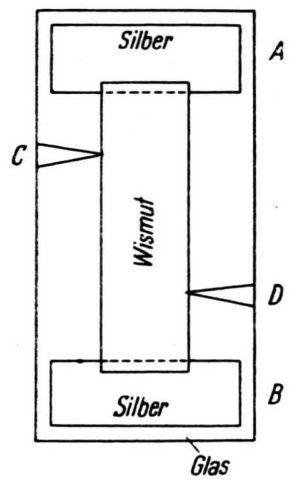

Abb. 1. Anordnung der Wismutschichten und Silberelektroden auf dem Glasträger.

Durch die Silberelektroden war die Kontaktierung der Wismutschicht mit den Zuleitungsdrähten und Thermoelementen erheblich erleichtert. Als Lot erwies sich eine Indiumlegierung mit einem Schmelzpunkt von etwas über $100^{\circ} \mathrm{C}$ als sehr geeignet.

Um von vornherein auszuschließen, daß die Meßergebnisse durch Verunreinigungen, die aus dem Schiffchenmaterial stammen, verfälscht wurden, haben wir Wismutschichten hergestellt, die aus Nickelschiffchen verdampft wurden. Trotz der guten Legierungsbildung im Zweiphasensystem $\mathrm{Bi}-\mathrm{Ni}$ konnte ein Einfluß von Nickelatomen in dickeren Aufdampfschichten, die noch keinen Weglängeneffekt zeigen, auf die Thermokraftmessungen außerhalb der Fehlergrenze von 1 bis $2 \%$ nicht festgestellt werden. Damit dürfte eine Verunreinigung der Wismutschichten mit Wolfram bei den niedrigen Aufdampftemperaturen das Meßergebnis erst recht nicht beeinflussen.

\section{Die Behandlung und Montierung der Schichten}

Zur Verhinderung einer oberflächlichen Oxydation und einer stärkeren Einlagerung von Gasmolekülen wurden die Glasträger mit den Schichten im Vakuum aufbewahrt. In Parallelversuchen hatte sich nämlich gezeigt, daß die differentielle Thermokraft der Wismutschichten gegen Kupfer je nach Schichtdicke um 10 bis 25\% abnimmt, wenn die Proben 24 Stdn. an Luft gelegen haben. Dabei ist der Einfluß auf die dünneren Schichten naturgemäß größer als auf die kompakteren.

Vor der Messung haben wir jede Probe 2 Stdn. bei $100^{\circ} \mathrm{C}$ im Hochvakuum getempert. Zur Bestimmung der Thermokraft waren die Glasplatten im Vakuum zwischen zwei Kupferklötzen eingekittet, die durch Thermostaten konstant auf verschiedener Temperatur gehalten werden konnten und so ein Temperaturgefälle in dem Träger erzeugten. Da die Wärmeleitung der Schicht klein gegenüber der des Glasträgers bleibt, ist das Temperaturgefälle fast ausschließlich durch letzteren bestimmt. Aus diesem Grunde haben wir die Elektrode C und D als Spitzen ausgebildet und die Thermoelemente zur Temperaturmessung unmittelbar am Schichtrande angebracht. Dadurch ist gewährleistet, daß die im Temperaturgefälle gemessene Spannungsdifferenz auch wirklich der zwischen C und D gemessenen Temperaturdifferenz zuzuordnen ist. Alle Spannungen wurden mit dem fünfstufigen thermokraftfreien Dießelhorstkompensator gemessen.

\section{Schichtdickenbestimmung und Meßergebnisse}

Größere Schwierigkeiten ergaben sich bei der Bestimmung der Schichtdicke. Als quantitatives Maß kann in erster Näherung der elektrische Widerstand zwischen den Elektroden $\mathrm{C}$ und D dienen, da deren Abstand und die Breite der Schicht bei allen Proben konstant gehalten wurden. So haben wir auch in der Abb. 2 die differentielle Thermospannung gegen Kupfer als Funktion dieses Widerstandes dargestellt. Es ergibt sich eine starke Abnahme der differentiellen Thermokraft, die bei den dünnsten Proben nur noch $14 \%$ des Wertes am kompakten Material erreicht.

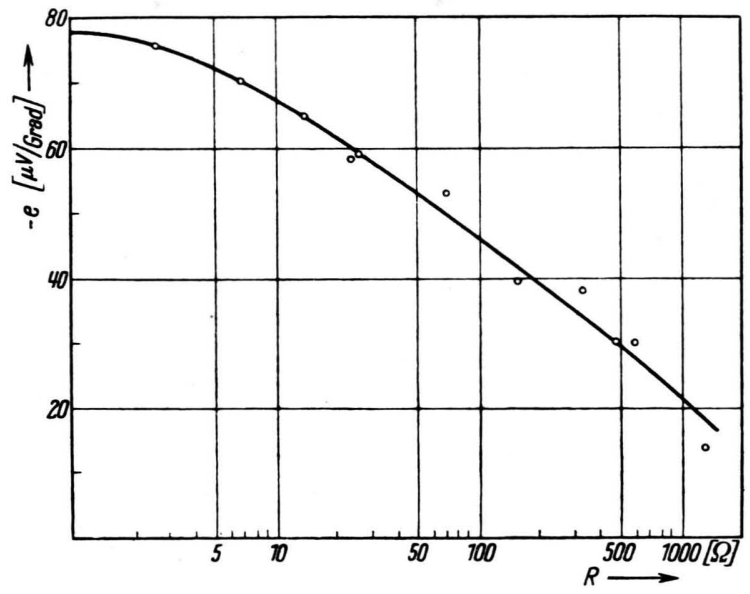

Abb. 2. Die differentielle Thermokraft von dünnen. BiSchichten gegen Kupfer als Funktion ihres elektrischen Widerstandes bei konstanter Schichtlänge und -breite.

Die größere Streuung der Meßpunkte bei den dünneren Schichten ist darauf zurückzuführen, daß einzelne Wismutkristallite bei verschiedener Orientierung gegeneinander Thermokräfte zeigen. Diese heben sich im polykristallinen Material im Mittel auf, während bei den geringeren Schichtdicken diese Mittelung mehr und mehr unvollständig wird und da- 


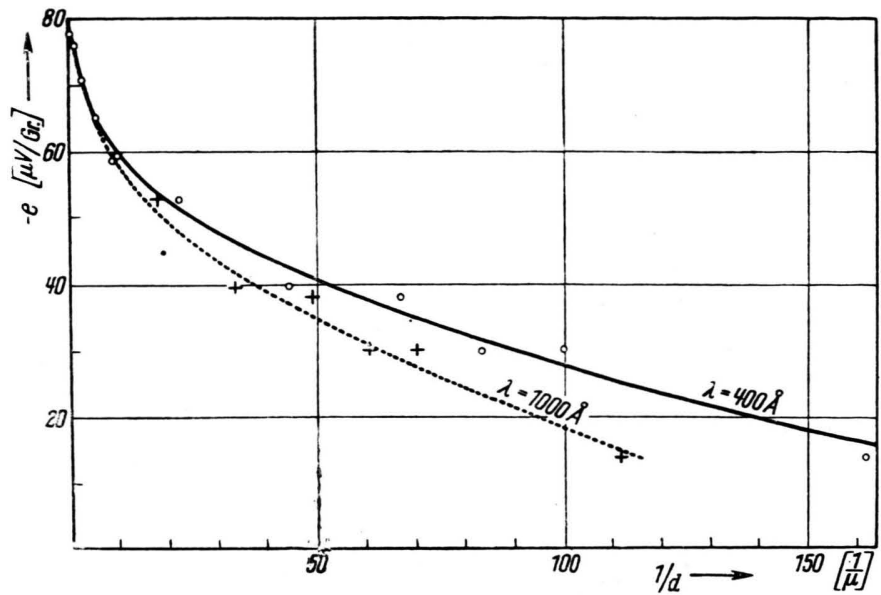

Abb. 3. Die differentielle Thermokraft von dünnen Wismutschichten gegen Kupfer in Abhängigkeit von der reziproken Schichtdicke.

durch die von Probe zu Probe wechselnde Kristallorientierung die Meßergebnisse verändert.

Um aus den gemessenen Widerstandswerten die reziproke Schichtdicke zu ermitteln, kann man theoretische Berechnungen von $\mathrm{Fuch} \mathrm{s}^{4}$ benutzen, der den Weglängeneffekt auf den elektrischen Widerstand dünner Metallfilme exakt berücksichtigt. Unter der Annahme der vollen Gültigkeit dieser Theorie lassen sich dann die Schichtdicken für einen bestimmten Wert der mittleren freien Weglänge errechnen. Nach Fuchs gilt:

$$
\begin{aligned}
& \frac{\varrho_{\infty}}{\varrho}=1+\frac{3}{4}\left[\frac{d}{\lambda}-\frac{1}{12}\left(\frac{d}{\lambda}\right)^{3}\right]\left(-E i\left(-\frac{d}{\lambda}\right)\right)-\frac{3}{8} \frac{\lambda}{d} \\
& \left(1-e^{-d / \lambda}\right)-\left[\frac{5}{8}+\frac{1}{16} \frac{d}{\lambda}-\frac{1}{16}\left(\frac{d}{\lambda}\right)^{2}\right] e^{-d / \lambda} \equiv f\left(\frac{d}{\lambda}\right) .
\end{aligned}
$$

Dabei sind $\varrho_{\infty}$ der spezifische elektrische Widerstand des kompakten Materials und $E i\left(-\frac{d}{i}\right)$ der Integrallogarithmus

$$
E i\left(-\frac{d}{\lambda}\right)=\int_{\infty}^{d / \lambda} \frac{e^{-x}}{x} \mathrm{~d} x .
$$

Damit folgt:

$$
R=\frac{l \underline{\varrho}}{b d}=\frac{l \varrho_{\infty}}{b d} \frac{1}{f\left(\frac{d}{i}\right)} \quad \begin{array}{r}
(b=\text { Breite }, \\
l=\text { Länge })
\end{array}
$$

oder

$$
\frac{1}{d}=\frac{R b}{l \varrho_{\infty}} f\left(\frac{d}{i}\right)
$$

${ }^{4} \mathrm{~K} . \mathrm{Fuchs}$, Proc. Cambridge Philos. Soc. 34, 100 [1938].
Aus dieser Beziehung ist $d$ für verschiedene $\lambda$ numerisch berechenbar. In der Abb. 3 haben wir die gemessenen Thermokräfte unter der Annahme von $\lambda=400 \AA$ und $\lambda=1000 \AA$ über den nach obigen Formeln bestimmten $1 / d$-Werten aufgetragen. Diese Weglängen liegen in der gleichen Größenordnung wie bei den anderen Metallen, obschon einige Experimente von Eucken und Mitarb. ${ }^{5}$ auf wesentlich höhere $\lambda$-Werte $(11 \mu$ !) führten. Da man außerdem die Widerstandsvermehrung an dünnen Schichten nicht allein auf den Weglängeneffekt zurückführen kann, erhält die Darstellung der differentiellen Thermokraft als Funktion der reziproken Schichtdicke nur einen qualitativen Charakter.

Für große Dicken konnte das $d$ auch durch Wägung der Wismutschicht ermittelt werden. Hier ergab sich eine Übereinstimmung mit den aus den Widerstandswerten ermittelten Größen. Bei den dünneren Schichten $(<0,5 \mu)$ hingegen versagte diese Methode. Aus diesem Grunde ist der Beginn des Einflusses der Schichtdicke auf die Thermokraft ziemlich genau festlegbar. So macht sich hier ein Effekt von 2\% erst bei $1 \mu$ bemerkbar, während bei $0,1 \mu$ schon $25 \% \mathrm{Ab}$ weichung vom kompakten Material resultieren.

In die gleiche Größenordnung für den Beginn des Schichtdickeneffektes kommt man mit der magnetischen Widerstandsänderung, die z. B. bei $0,1 \mu$ dicken Proben bei Zimmertemperatur und $25000 \mathrm{Gau}$ nur $6 \%$ gegenüber etwa $125 \%$ beim kompakten Material beträgt. Zur Erklärung dieser Abweichung würde eine Vergrößerung des Widerstandes um den Faktor 5 ausreichen, der seinerseits aber auf eine Weglänge von $1 \mu$ führen würde ${ }^{4}$.

\section{Diskussion der Ergebnisse}

Zur Deutung dieser Ergebnisse kann man gerade beim Wismut verschiedene Wege vorschlagen. Will man den ganzen Effekt auf die mittlere freie Weglänge zurückführen, so muß man für diese wenigstens den unwahrscheinlich hohen Wert von $10000 \AA$ annehmen. Von Experimenten an reinstem Mylius-Gold weiß man aber, daß der Weglängeneffekt zur Deutung der Widerstandsvermehrung an dünnen Proben um den Faktor 3 bis 5 nicht ausreicht. Hier können Oberflächeneinflüsse entscheidend mitwirken ${ }^{6}$. Ließen wir eine ähnliche Diskrepanz auch für die Thermo-

5 A. E u ck e n u. F. F ör s t e r, Ges. Wiss. Göttingen, math.-physik. Kl. 1, 43, 129 [1934].

6 E. Justi, M. Kohler u. G. Lautz, Abh. Braunschw. Wiss. Ges. 3, 34 [1951]. 
kraft des Wismut zu, würde sich ein Wert von 3000 bis $2000 \AA$ ergeben.

Weitere Komplikationen treten durch die Eigenart des Leitungsmechanismus im festen Wismut auf. Bekanntlich ist die Elektronenleitung durch die sehr schwache Überlappung einer voll besetzten und einer leeren Brillouin-Zone möglich. Diese Überschneidung beträgt etwa $10^{-3}$ eVolt. Dadurch liegt die FermiEnergie in der Nähe zweier Bandränder, und es ergibt sich eine sehr kleine scheinbare Elektronenmasse von $0,01 m_{0}\left(m_{0}=9,03 \cdot 10^{-28} \mathrm{~g}\right)$. Das als Freiheitszahl definierte Verhältnis

$$
f=\frac{m_{0}}{m}
$$

erreicht den großen Wert von annähernd 100. Wie wir an anderer Stelle schon angedeutet haben, ist es vielleicht zweckmäßiger, bei diesen dünnen Schichten den Gitteraufbau in einer Dimension exakt zu berücksichtigen ${ }^{6}$. Dann dürfte man die Metallfilme bei der Berechnung ihrer thermischen und elektrischen
Eigenschaften nur noch in zwei Dimensionen als Quasikontinuum betrachten. Unter diesen Annahmen erhält man in erster Näherung eine Veränderung der Fermi-Energie um 1\%, wenn die Schichtdicke etwa $1000 \AA$ wird. Das würde aber bei einer Bandüberlappung von nur $10^{-3} \mathrm{eVolt}$ eine Änderung der scheinbaren Masse und der effektiven Elektronenzahl um wenigstens $100 \%$ bedingen. Da in die Formeln meist $m / \lambda$ eingeht, ließe sich eine Vergrößerung der scheinbaren Masse formal auch durch eine Verkleinerung der mittleren freien Weglänge bei konstant gehaltenem $m$ erfassen. Man darf also folgern, daß die $\lambda$ Werte beim Wismut nicht eindeutig aus den Experimenten ablesbar sind, sondern auch Änderungen in der Bindung der Elektronen einen ähnlichen Effekt bewirken können.

Schließlich haben wir bei allen Betrachtungen die Oberflächenzustände außer acht gelassen, die bei der Statistik der Elektronen für dünne Schichten mehr und mehr zu berücksichtigen sein werden. Auch diese können einen Schichtdickeneffekt bedingen, der nicht mit der freien Weglänge direkt zusammenhängt.

\title{
Erfahrungen mit Metallfolien als Hochvakuumdichtungen
}

\author{
Von Heinrich Hintenberger * \\ Aus dem Max-Planck-Institut, Mainz \\ (Z. Naturforschg. 6 a, 459-462 [1951]; eingegangen am 9. Juni 1951)
}

\begin{abstract}
Es werden die Erfahrungen mit lösbaren und ausheizbaren, vakuumdichten Flanschverbindungen beschrieben, bei denen Metallbleche, insbesondere Aluminiumbleche, als Dichtungsfolien Verwendung finden. Form und Dimensionierung der Flansche und die Behandlung der Dichtungsfolien, die sich bei zuverlässigen Dichtungen in jahrelangem Betrieb bewährt haben, werden angegeben. Aluminiumbleche zwischen Messingflanschen können bis $280^{\circ} \mathrm{C}$ erhitzt werden, ohne undicht zu werden.
\end{abstract}

$I$ ösbare, hochvakuumdichte Verbindungen, bei denen Blei, Zinn, Aluminium, Kupfer oder Silber zur Dichtung verwendet werden, sind mehrfach in der Literatur erwähnt und z. Tl. auch näher erläutert worden. So beschreiben Es pe und K n o $11^{1}$ eine Flanschabdichtung mit Aluminium und Blei, Strong ${ }^{2}$ verwendet Bleidrähte, $\mathrm{S}$ h o u p p ${ }^{3}$ Zinndrähte, $\mathrm{B}$ u s h ${ }^{4}$ Kupferringe und $\mathrm{Pau}^{5}$ Silberfolien $^{6}$ zur Herstel-

* Z. Zt. als Gast am Theodor-Kocher-Institut der Universität Bern.

1 W. Espe u. M. Knoll, „Werkstoffe der Hochvakuumtechnik“, Berlin 1936, S. 322. M. K n o l1, Z. techn. Physik 10, 294 [1929].

2 J. S t r o n g, „Modern Laboratory practice“, 15. Aufl. Blackie \& son, London 1949, S. 128. lung vakuumdichter Verbindungen. Besonders bei ausheizbaren Massenspektrometern wurden in den letzten Jahren zuweilen Metalldichtungen angewendet. $\mathrm{King}$ don und $\mathrm{Pollock}{ }^{7}$ benutzen Bleidichtungen, $\mathrm{B} \mathrm{leakney}$ und $\mathrm{Cumings}{ }^{8}$ Aluminium-

3 S h o u p p, Res. Report, Westinghouse Lab.; L. H. M a rtin u. R. D. Hill, „Vacuum Practice“, Univ. Press, Melbourne 1947, S. 67.

4 W. E. Bush in A. Gutherie u. R. K. Wakerling, „Vacuum Equipment and Techniques“, Mc GrawHill Book Co., New York 1949, S. 161.

5 W. P a u l, Z. Physik 124, 244 [1948].

6 R. J ä ck e l, „Kleinste Drucke“, Springer, Berlin 1950, S. 248.

7 K. H. King do n u. H. C. Pollock, Physic. Rev. 57, 1072 [1940]. 